\title{
Brief report: Plasma oxytocin is lower in children with Asperger syndrome and associated with autistic trait attention to detail
}

\author{
Veronika Husarova $^{1,2}$, Silvia Lakatosova ${ }^{1}$, Anna Pivovarciova ${ }^{1}$, Jan Bakos ${ }^{1}$, Jaroslava Durdiakova ${ }^{1}$, \\ Aneta Kubranska ${ }^{1}$, Daniela Ostatnikova ${ }^{1}$ \\ ${ }^{1}$ Institute of Physiology, Medical Faculty Comenius University, Bratislava, Slovakia \\ ${ }^{2}$ Clinic of Psychiatry, Martin University Hospital and Jessenius Faculty of Medicine, Comenius University, Martin, Slovakia \\ Email: veronika.husarova@fmed.uniba.sk
}

Received 3 September 2013; revised 1 October 2013; accepted 9 October 2013

Copyright (C) 2013 Veronika Husarova et al. This is an open access article distributed under the Creative Commons Attribution License, which permits unrestricted use, distribution, and reproduction in any medium, provided the original work is properly cited.

\begin{abstract}
Aim: Autism spectrum disorders (ASD) are described as a continuum of severity gradient of autistic symptoms diffusing through particular ASD diagnoses, however the biological correlates among individuals with the different ASD diagnoses slightly or considerably differ. Oxytocin (OT) has been implicated to play an important role in autism etiology. Lower OT levels have been previously found in children with infantile autism, however in a group of high-functioning autistic subjects, no differences have been shown compared to controls. Moreover, whereas the opposite patterns of $\mathrm{OT}$ associations with social measures have been found in children with infantile autism compared to healthy children, no associations have been found in individuals with high-functioning autism. We aimed to find out the plasma $O T$ differences between separate group of children with Asperger syndrome (AS) and healthy children and the associations of OT with particular autistic traits in a group of children with AS. Methods: We included 9 children $(m=6, f=3)$ with AS at the age 9 to 12 years and 9 age- and gender-matched controls. OT levels were analyzed by ELISA method. Autistic traits in children with AS were evaluated by Autism Spectrum Quotient (AQ), child and adolescent versions. Results: Children with AS had significantly lower plasma OT levels compared to healthy children. We found the significant negative correlation of OT level and AQ Attention to detail area score. Conclusion: In spite of the lower OT level in children with AS, which is also previously found in children with infantile autism, the pattern of OT associations with autistic traits more
\end{abstract}

resembles the pattern in non-autistic population. Our preliminary results support the hypothesis of continuum within the ASD particular diagnoses in the terms of biological correlates.

Keywords: Oxytocin; Asperger Syndrome; Autism Spectrum Quotient

\section{INTRODUCTION}

Asperger syndrome (AS) is an autism spectrum disorder (ASD) with symptoms including difficulties in understanding the rules of social interaction, lack of empathy, stereotyped or unusual behavior, interests or activities with no history of formal speech delay and with normal or even above-average intelligence [1]. It is often described as a milder form of autism with the traits situated in a continuum of severity gradient of autistic symptoms [2], however the neurobiological correlates among individuals with AS and autism slightly or considerably differ [3].

Oxytocin (OT) has been implicated to play an important role in etiology of ASD due to its effects on emotional and social behavior [4,5]. Children with autism had significantly lower plasma OT levels than the control group [6,7] with the different pattern of OT associations to social and developmental measures [6]. However, no differences in OT level and no associations between OT level and autistic symptoms have been found in a separate group of high-functioning autistic subjects compared to controls [8]. Despite the evidence of improvement of social [9] and repetitive [10] behavior after OT administration in individuals also with AS and the finding of the OT receptor (OXTR) role in etiology of high-functioning 
level autistic disorders including AS [11], to our best knowledge, no study has evaluated the plasma OT levels in a separate group of children with AS compared to healthy controls. We aimed to find out the differences in plasma OT levels in the group of children with AS compared to healthy age- and gender-matched controls and the associations of plasma OT levels with particular autistic traits.

\section{MATERIALS AND METHODS}

\subsection{Participants}

We included 9 children (males $=6$, females $=3$ ) with the diagnosis of Asperger syndrome and 9 healthy age- and gender-matched control children. The AS group age was: mean $=10.25, \mathrm{SD}=1.39$ years; the control group age was: mean $=10.63, \mathrm{SD}=1.85$ years. Children with AS were recruited from the local Autism Centre for Children in Bratislava, Slovakia. All children with AS were diagnosed as meeting criteria for ICD-10 Asperger syndrome by a clinical child psychologist with the cooperation of a child psychiatrist, who confirmed all diagnoses.

Control subjects were recruited through local pediatrician ambulance after regular pediatric examination or mild physical condition due to which they attended pediatrician. All subjects were at the time of blood sample collection healthy and without any known physical condition that was confirmed by pediatrician. All control subjects were without any psychiatric condition confirmed by child psychiatrist according to their examinations and parent interview. All control subjects had normal functioning in an elementary school. No control subject had delay in speech and language or psychomotor development as per parent interview. All subjects were medication-free.

All procedures were carried out with the adequate understanding of participants. Written informed consent was obtained from all parents of participating children. The protocol was approved by the Ethics Committee of Faculty of Medicine, Comenius University. The study conformed to the code of ethics stated in the Declaration of Helsinki.

\subsection{Plasma Oxytocin Measurement}

Three $\mathrm{mL}$ of venous blood were taken from children with AS in the local Neurological Centre for Children and Adults in Bratislava. Blood of control children was taken by their pediatrician. Immediately after samples intake, blood was centrifuged. After 10 minutes centrifugation at 3000 RPM, plasma was taken with addition of protease inhibitor aprotinin (Sigma Aldrich). Plasma samples with aprotinin were stored at $-20^{\circ} \mathrm{C}$ until OT measurement. Plasma OT levels were measured using ELISA method according to manufacturer's instructions (Enzo Life Sci- ences). All OT measurements were performed in the laboratory of Institute of Physiology, Comenius University in Bratislava.

\subsection{Psychological Measures in Children with AS}

Mothers of children with AS were asked to complete the Autism spectrum quotient questionnaire, children's version (AQ-child), the adolescent version was used in one 12-year-old boy. The AQ-child is parent-report questionnaire to detect autistic traits in children at $4-11$ years, adolescent version is used for children at $12-15$ years of age. Both versions consist of 50 items including 5 areas associated with autism or BAP (broader autism phenotype): social skill, attention switching, attention to detail, communication and imagination, each represented by 10 items. The higher score represents the greater autistic traits. In AQ-child parents can "definitely agree", "slightly agree", "slightly disagree" and "definitely disagree" with the statements about their child, with the following answer categories: 0 representing definitely agree; 1 slightly agree; 2 slightly disagree; and 3 definitely disagree. Items are reverse scored as necessary. Maximum score is 150 , the cut-off score is 76 . We used the same 4-point scale also in the AQ-adolescent version used in 1 boy. Both scales have been shown to have a good test-retest reliability and internal consistency [12, 13].

AQ questionnaires were downloaded from Autism Research Centre website

http://www.autismresearchcentre.com/ with kind permission of the authors and translated into Slovak language.

\subsection{Statistical Analysis}

We used the two-tailed unpaired t-test for group differences in plasma OT levels between children with AS and healthy controls. We used Pearson correlation coefficients to correlate plasma OT levels with AQ total score and AQ subscale scores. The significance level was set at $\mathrm{p}<0.05$.

\section{RESULTS}

\subsection{Plasma Oxytocin Levels Group Differences}

We found a statistically significant difference in plasma OT levels between groups $(t=2.27, p=0.037)$. Descriptive statistics and group differences for OT levels are presented in Table 1.

\subsection{Plasma Oxytocin Levels and Particular AQ Subscales}

We found the significant negative correlation of plasma OT levels and attention to detail area of AQ. We did not find the significant correlations of plasma OT levels and 
Table 1. Plasma oxytocin levels in children with Asperger syndrome and controls $(\mathrm{pg} / \mathrm{mL})$.

\begin{tabular}{ccc}
\hline & Children with AS $(\mathrm{n}=9)$ & Controls $(\mathrm{n}=9)$ \\
\hline Mean (SD) & $155.52(110.00)$ & $320.64(188.27)^{*}$ \\
Median & 151.08 & 284.17 \\
Range & $32.40-279.13$ & $58.61-748.49$ \\
\hline
\end{tabular}

${ }^{*} \mathrm{t}=2.27, \mathrm{p}=0.037$.

any other AQ subscale or AQ total score. Descriptive statistics of AQ scores and correlations with plasma OT levels are presented in Table 2.

\section{DISCUSSION}

To our best knowledge, this is the first study evaluating OT levels and its associations with particular autistic traits in children with Asperger syndrome exclusively. We found significantly lower plasma OT levels in children with AS compared to control age- and gendermatched group. Previous studies have shown decreased OT levels in children with infantile autism [6,7], however no differences have been found in children with high-functioning autism [8]. Moreover, plasma OT levels seem to be differently associated with autism symptoms within the different ASD levels. Whereas in children with infantile autism, OT levels have been associated with lower scores on social and developmental measures [6], in a separate group of high-functioning autistic subjects, no effects of OT level on particular symptoms have been found [8]. On the contrary, in a group of healthy children, the OT levels have been associated with better social skills [6]. In our study of children with AS exclusively, we found the negative association between OT levels and AQ attention to detail area, thus the higher OT level was associated with lower autistic traits similarly to healthy children [6]. This indicates that in spite of the lower OT level in children with AS, the pattern of OT associations with autistic traits resembles the nonautistic population. However, we found only the association of $\mathrm{OT}$ and AQ attention to detail area. We did not find the OT associations with the social skill or communication areas indicating that endogenous OT could differently affect social measures in healthy and AS individuals.

Our results of decreased OT, like in children with infantile autism, with the similar pattern of OT associations and with particular autistic traits to non-autistic individuals, support the hypothesis of ASD continuum in the terms of biological correlates. The further studies should compare the OT levels and its associations with behavioral measures separately in children with the diagnoses of infantile autism, atypical autism and Asperger syndrome (ICD-10) or the different levels of Autism Spectrum Disorders (DSM-V).
Table 2. Descriptive statistics of AQ areas of children with Asperger syndrome and Pearson correlations with oxytocin level.

\begin{tabular}{lccc}
\hline & Mean (SD) & Pearson $\mathrm{r}$ & $\mathrm{p}$ value \\
\hline AQ total score & $94.0(12.4)$ & -0.26 & 0.566 \\
AQ social skill & $20.6(2.9)$ & 0.01 & 0.977 \\
AQ attention switching & $18.7(6.5)$ & -0.35 & 0.445 \\
AQ attention to detail & $18.9(4.2)$ & -0.77 & $0.045^{*}$ \\
AQ communication & $20.9(4.1)$ & 0.24 & 0.599 \\
AQ imagination & $15.0(5.7)$ & 0.21 & 0.656 \\
\hline
\end{tabular}

${ }^{*}$ Statistically significant result.

Limitations of the study include small number of subjects in both groups and the fact that males and females were not separated. Gender has been found to have effect on OT level in the overall group of autistic and non-autistic children and OT associations with anxiety [8]. However, as groups in our study were also gendermatched, we do not expect the different finding in plasma OT levels group differences after gender separation.

Furthermore, we used only AQ for behavioral assessment of children. AQ was designed to evaluate the different areas of autistic traits, however, it is restricted to them. As OT has been found to be associated with anxiety in a group of autistic and non-autistic girls [8], more extensive behavioral assessment of emotional and social functioning should be used in further studies. Our preliminary results should be followed by studies with higher number of subjects including the different ASD diagnoses with gender separation.

\section{ACKNOWLEDGEMENTS}

Authors thank to all parents and their children for participation in the study. The study was supported by the following grants: APVV-025411, APVV-0253-10, VEGA-1/0066/12 and UK 67/2013.

\section{REFERENCES}

[1] Carpenter, L.A., Soorya, L. and Halpern, D. (2009) Asperger's syndrome and high-functioning autism. Pediatric Annals, 38, 30-35. http://dx.doi.org/10.3928/00904481-20090101-01

[2] Spiker, D., Lotspeich, L.J., Dimiceli, S., Myers, R.M. and Risch, N. (2002) Behavioral phenotypic variation in autism multiplex families: Evidence for a continuous severity gradient. American Journal of Medical Genetics, 114, 129-136. http://dx.doi.org/10.1002/ajmg.10188

[3] Yu, K.K., Cheung, C., Chua, S.E. and McAlonan, G.M. (2011) Can Asperger syndrome be distinguished from autism? An anatomic likelihood meta-analysis of mri studies. Journal of Psychiatry \& Neuroscience: JPN, 36, 412421.

[4] Neumann, I.D. (2008) Brain oxytocin: A key regulator of 
emotional and social behaviours in both females and males. Journal of Neuroendocrinology, 20, 858-865. http://dx.doi.org/10.1111/j.1365-2826.2008.01726.x

[5] Stavropoulos, K.K. and Carver, L.J. (2013) Research review: Social motivation and oxytocin in autism-implications for joint attention development and intervention. Journal of Child Psychology and Psychiatry, and Allied Disciplines, 54, 603-618. http://dx.doi.org/10.1111/jepp.12061

[6] Modahl, C., Green, L., Fein, D., Morris, M., Waterhouse, L., Feinstein, C. and Levin, H. (1998) Plasma oxytocin levels in autistic children. Biological Psychiatry, 43, 270277. http://dx.doi.org/10.1016/S0006-3223(97)00439-3

[7] Al-Ayadhi, L.Y. (2005) Altered oxytocin and vasopressin levels in autistic children in central Saudi Arabia. Neurosciences, 10, 47-50.

[8] Miller, M., Bales, K.L., Taylor, S.L., Yoon, J., Hostetler, C.M., Carter, C.S. and Solomon, M. (2013) Oxytocin and vasopressin in children and adolescents with autism spectrum disorders: Sex differences and associations with symptoms. Autism Research: Official Journal of the International Society for Autism Research, 6, 91-102.

[9] Hollander, E., Bartz, J., Chaplin, W., Phillips, A., Sumner, J., Soorya, L., Anagnostou, E. and Wasserman, S. (2007) Oxytocin increases retention of social cognition in autism. Biological Psychiatry, 61, 498-503. http://dx.doi.org/10.1016/j.biopsych.2006.05.030

[10] Hollander, E., Novotny, S., Hanratty, M., Yaffe, R., DeCaria, C.M., Aronowitz, B.R. and Mosovich, S. (2003) Oxytocin infusion reduces repetitive behaviors in adults with autistic and asperger's disorders. Neuropsychopharmacology: Official Publication of the American College of Neuropsychopharmacology, 28, 193-198.

[11] Wermter, A.K., Kamp-Becker, I., Hesse, P., SchulteKorne, G., Strauch, K. and Remschmidt, H. (2010) Evidence for the involvement of genetic variation in the oxytocin receptor gene (oxtr) in the etiology of autistic disorders on high-functioning level. American Journal of Medical Genetics Part B, Neuropsychiatric Genetics: The Official Publication of the International Society of Psychiatric Genetics, 153B, 629-639.

[12] Baron-Cohen, S., Hoekstra, R.A., Knickmeyer, R. and Wheelwright, S. (2006) The autism-spectrum quotient (aq) -Adolescent version. Journal of Autism and Developmental Disorders, 36, 343-350. http://dx.doi.org/10.1007/s10803-006-0073-6

[13] Auyeung, B., Baron-Cohen, S., Wheelwright, S. and Allison, C. (2008) The autism spectrum quotient: Children's version (aq-child). Journal of Autism and Developmental Disorders, 38, 1230-1240. http://dx.doi.org/10.1007/s10803-007-0504-Z 